\title{
Beyond cognitive myopia: a patchwork approach to the concept of neural function
}

\author{
Philipp Haueis
}

Berlin School of Mind and Brain

Humboldt Universität zu Berlin

Luisenstr. 56

10117 Berlin, Germany

Abteilung Philosophie

Universität Bielefeld

Postfach 100131

33501 Bielefeld, Germany

Email: philipp.haueis@uni-bielefeld.de

ORCID: 0000-0002-0935-9015

ABSTRACT: In this paper, I argue that looking at the concept of neural function through the lens of cognition alone risks cognitive myopia: it leads neuroscientists to focus only on mechanisms with cognitive functions that process behaviorally relevant information when conceptualizing "neural function". Cognitive myopia tempts researchers to neglect neural mechanisms with noncognitive functions which do not process behaviorally relevant information but maintain and repair neural and other systems of the body. Cognitive myopia similarly affects philosophy of neuroscience because scholars overlook noncognitive functions when analyzing issues surrounding e.g., functional decomposition or the multifunctionality of neural structures. I argue that we can overcome cognitive myopia by adopting a patchwork approach that articulates cognitive and noncognitive "patches" of the concept of neural function. Cognitive patches describe mechanisms with causally specific effects on cognition and behavior which are likely operative in transforming sensory or other inputs into motor outputs. Noncognitive patches describe mechanisms that lack such specific effects; these mechanisms are enabling conditions for cognitive functions to occur. I use these distinctions to characterize two noncognitive functions at the mesoscale of neural circuits: subsistence functions like breathing are implemented by central pattern generators and are necessary to maintain the life of the organism. Infrastructural functions like gain control are implemented by canonical microcircuits and prevent neural system damage while cognitive processing occurs. By adding conceptual patches that describe these functions, a patchwork approach can overcome cognitive myopia and help us explain how the brain's capacities as an information processing device are constrained by its ability to maintain and repair itself as a physiological apparatus.

KEYwORDS: Neural Function; Cognitive Myopia; Maintenance and Repair; Causal Specificity; Concepts; Patchwork 
We must actively frame semantic pictures if we hope to improve our usage through other means than brute trial and error, but it is easily possible to lean upon portraits that are quite badly mistaken or shortsighted.

Mark Wilson

\section{Introduction}

What are the functions of the central nervous system (CNS) and how do neuroscientists conceptualize them? Consider the following general takes:

A major task of the CNS is to configure the way in which sensory information becomes linked to adaptive responses and meaningful experiences (Mesulam 1998, p. 1014).

If we could observe or feel the brain at work, it would be immediately obvious that neuronal function is as related to how we see, interpret, and react, as muscle contractions are related to the movements we make. (Llinas 2002, pp. 4).

Today it is possible to link the molecular dynamics of individual nerve cells to representations of perceptual and motor acts in the brain and to relate these internal mechanisms to observable behavior. (Kandel et al. 2000, pp. 3-4).

[The] dream of neurobiology [is] to understand all aspects of interesting and important cognitive phenomena-like memory - from the underlying molecular mechanisms through behavior (Stevens 1996, pp. 1147).

These quotes suggest that in subfields that study the function and dysfunction of neural systems - such as sensory and motor physiology, molecular neurobiology of memory, behavioral neurology, biological psychiatry—neuroscientists frequently conceptualize "neural function" in the vocabulary of cognition. They try to understand how neural entities and activities contribute to external behavior and internal experiences by processing sensory information (Mesulam). The brain's function in cognition is as obvious as the muscles' function in movement, save for the epistemic obstacles (Llinas). To understand cognitive CNS functions, the neurosciences must lift those epistemic obstacles and study the neural mechanisms of perception, action, learning and memory (Kandel, Stevens). Philosophers have followed such neuroscientists in their focus on cognitive CNS functions. Bickle (2003, p. 3) takes Kandel et al.'s quote to argue that neuroscientific explanations of cognition and behavior are reductionist, while Craver (2007, p. 168) takes Stevens' quote to argue that such explanations span multiple levels. Echoing Mesulam and Llinas, Bechtel (2008) argues that neuroscientists often aim to discover and explain "mental mechanisms" that process information relevant to the behavior of the organism. These scholars seem to share the intuition that because the brain is the "organ of thought", neural functions are best conceptualized in terms of cognition and behavior. 
In this paper, I argue that the vocabulary of cognition should be understood as one among many useful perspectives on the concept of "neural function". If we do not take these other perspectives into account, we risk what I call cognitive myopia:

Cognitive myopia: A given description is an instance of "neural function" if and only if it characterizes a set of neural entities and activities as processing information that directly contributes to one or several cognitive and behavioral capacities of the organism.

The above definition captures an implicit tendency that is exhibited by all those neuroscientists and philosophers who predominantly or exclusively focus on cognitive functions when conceptualizing "neural function". Cognitive myopia does not affect all neuroscientists, since many do not study neural mechanisms related to cognition and behavior at all. Yet it is prevalent and problematic enough in systems neuroscience contexts and philosophical circles to warrant explicit analysis. ${ }^{1}$ Cognitive myopia proceeds from the claim that the CNS contributes to all cognitive and behavioral capacities of an organism to the implicit conclusion that all mechanisms that "neural function" refers to are best conceptualized as contributing to cognition and behavior. While the former assumption is certainly reasonable and most likely true, the latter is likely unreasonable and almost certainly false. The CNS also does many things besides cognition: it regulates physiological parameters (e.g., blood pressure, glucose levels, arousal, sleep and wake cycle) and it maintains its structural integrity by constantly rebuilding ion channels, membranes and synaptic connections (Marder and Goaillard 2006, Squire et al. 2012, section IV). I call such neural maintenance and repair processes noncognitive functions:

Noncognitive functions: Activities of neural entities that (i) do not directly contribute to behavior and cognition by processing behaviorally relevant information because they (ii) contribute to structural integrity and homeostatic equilibrium by maintaining and repairing neural systems in the CNS or other systems within the body.

This biconditional definition captures three ways in which cognitive myopia can hinder the study of noncognitive functions. It can lead researchers to simply overlook such functions because it does not count descriptions that fulfill condition (ii) as instances of "neural function". Or it can tempt researchers to shoehorn noncognitive functions into cognitive vocabulary although they fulfil condition (i). An example is the tendency to interpret endogenous neural activity as directly contributing to cognition (Yuste et al. 2005, Bechtel 2013). Cognitive myopia can also tempt researchers to discard entities and activities with noncognitive functions as nonfunctional altogether because they fulfil conditions (i) and (ii). Examples of the latter

\footnotetext{
${ }^{1}$ Perhaps cognitive myopia is more problematic in philosophy of neuroscience than in neuroscientific practice as a whole. Philosophers often narrowly focus on cognitive, computational and behavioral neuroscience research. An exception is Craver and Robins (2009) and Bechtel and Abrahamsen (2009) on biological clocks.
} 
tendency are the characterization of task-independent fluctuations as functionally insignificant "noise" in early neuroimaging research (Raichle 2015), or the characterization of gamma oscillations as an "epiphenomenon" because they do not contribute to visual processing (Ghose and Freeman 1992). Cognitive myopia hinders discovery by preventing neuroscientists from exploring all possible functions of such activities. By narrowing the search space to information processing mechanisms, cognitive myopia also leads to impoverished explanations of cognitive capacities. Information processing requires many physiological operations in the CNS that keep the organism in homeostatic equilibrium (Marder and Goiallard 2006, Engl and Atwell 2015). Cognitive myopia prevents researchers from trying to explain how cognitive functions fit into this broader neurophysiological context (cf. Bechtel 2008, pp. 225f.). It hinders them from seeing that understanding the CNS as a cognitive machine requires understanding it as a biological organ. This point is crucial because it shows that the problem cannot be sidestepped by dividing the epistemic labor between say, cognitive neuroscientists studying cognitive functions and neurobiologists studying noncognitive ones. Even researchers who primarily care about cognitive functions should not background noncognitive functions because they both enable and constrain the kinds of cognitive function that the CNS actually supports.

Besides hindering empirical research, cognitive myopia also affects various issues in philosophy of neuroscience. Philosophical analyses of resting state neuroimaging research exhibit cognitive myopia: they exclusively focus on its role in revising models of cognitive architecture (Bechtel 2013), measuring long-term mental processes (Klein 2014), or drawing psychological inferences (McCaffrey and Danks 2017). Similarly, accounts of functional decomposition in neuroscience predominantly focus on the localization of cognitive capacities in structures of the CNS (Bechtel 2008 chs. 2 \& 3, Bechtel and Richardson 2010, chs. 4 \& 6). They thereby suggest that successful functional decomposition describes the cognitive functions of a neural structure, while its noncognitive functions can be backgrounded. Philosophers who discuss the problem of multifunctionality reinforce this exclusive focus on cognitive function. Philosophical accounts of this problem aim to show how functional localization is possible when a neural structure contributes to different cognitive and behavioral tasks in different contexts (Rathkopf 2013, McCaffrey 2015, Burnston 2016). These accounts exhibit cognitive myopia because they implicitly assume that "multifunctionality" means "cognitive multifunctionality". Cognitive myopia prevents philosophers from including noncognitive functions into their accounts of functional decomposition or multifunctionality. Even philosophers who primarily care about cognitive functions should not background 
noncognitive functions because they constrain adequate explanations of cognitive functions and are required to situate cognitive functions within the neural infrastructure that enables it.

In this paper, I argue that we can overcome cognitive myopia by adopting a patchwork approach to the concept "neural function". Rather than imposing pre-conceived philosophical theories of concepts onto scientific examples, patchwork approaches focus on the material inferential structure of scientific concepts as they are actually used in practice (Wilson 2006, Love 2013, Bursten 2016, Novick 2018). ${ }^{2}$ Scientific concepts develop a patchwork of local applications when practitioners use concepts to refer to related but non-identical properties when extending them to novel cases in their domain of inquiry. Such differences are not reflected immediately in the semantic pictures of a concept, which "embody the generic stories that speakers tell themselves with respect to how their predicate's usage matches to worldly support within normal circumstances of application.” (Wilson 2006, p. 516). By recognizing the patchwork structure of how a concept is used, scientists can and frequently do correct errors in their semantic pictures (see ibid. for historical examples).

I contend that cognitive myopia provides neuroscientists and philosophers with an erroneous semantic picture of "neural function" because it suggests that this concept univocally refers to cognitive mechanisms. We can overcome this picture once we recognize that the conceptual structure of "neural function" consists of several "patches" that describe either cognitive or noncognitive kinds of function. In this paper, I articulate three such patches by analysing neural functions at the mesoscale. This scale consists of neural circuits with a few 100 to $\sim 10.000$ cells. Examples of entities at the mesoscale are central pattern generator circuits in invertebrates, the spinal cord and brain stem, or microcircuits of synaptically interconnected neurons in the cortex (Grillner et al. 2005). A patchwork approach helps neuroscientists to better understand what these circuits do because they can contribute to either noncognitive functions (central pattern generators) or both cognitive and noncognitive functions (cortical microcircuits). By adding noncognitive kinds of function, the patchwork model developed below helps us rethink the taxonomy of mesoscale functions beyond a cognitively myopic picture of "neural function". Investigating noncognitive functions is crucial both to understand the maintenance and repair mechanisms that are specific to neural systems and to understand the constraints on information processing at the very same scale at which given cognitive

\footnotetext{
2 Patchwork approaches share this broadly naturalistic approach to concepts with a growing number of philosophers that focus on the practical roles that concepts play in scientific measurement (Chang 2004), problem solving (Nersessian 2008) experimentation and modeling (see Feest and Steinle 2012 and Rouse 2015).
} 
function is being studied. By using noncognitive patches of "neural function" to characterize these constraints, researchers can understand the enabling conditions of the information processing mechanisms that cognitive patches of this concept refer to.

Sect. 2 motivates the claim that the concept "neural function" exhibits a patchwork structure. Cognitive patches of this concept refer to mechanisms with causally specific effects on cognition and behavior, while noncognitive patches refer to mechanisms without such specific effects. In sect. 3, I use this distinction to articulate two noncognitive patches of "neural function". Subsistence functions like breathing are implemented by central pattern generators and maintain the life of the organism. Infrastructural functions like gain control are implemented by cortical microcircuits and prevent neural system damage while cognitive processing occurs. In sect. 4, I use infrastructural and subsistence patches to articulate a tripartite patchwork model that tracks how different kinds of circuit function are distributed at the mesoscale. This model helps researchers overcome cognitive myopia because it provides empirical and conceptual tools to study kinds of neural circuit that are not adequately described as being cognitive. I conclude by showing how a patchwork approach can be extended from mesoscale research to other neuroscientific subfields and philosophical research on neural functions.

\section{Cognitive and noncognitive patches of "neural function"}

In this section, I motivate the claim that the concept "neural function" exhibits a patchwork structure. I show why "neural function" has different local applications depending on which experimental methods and vocabulary neuroscientists use to search for neural mechanisms. These local applications render the meaning of "neural function" differently. Each local application domain plus methods and vocabulary corresponds to a segment or "patch" in the patchwork structure of "neural function". Cognitive patches of "neural function" refer to information processing mechanisms with causally specific effects on cognition and behavior, whereas noncognitive patches refer to maintenance and repair mechanisms that lack such specific effects.

The sense of "neural function" that is characteristic of cognitive patches of this concept is expressed well by deCharms and Zado (2000, p. 614): "Function is the effect the [neural] signal can have on cognitive processes and resultant behavior, e.g. the function of a neuron's 
spikes in triggering an eye-blink reflex". When neuroscientists render the meaning of "neural function" this way to search for neural mechanisms, they typically conceptualize these mechanisms as processing information that is relevant to the behavior of the whole organism (Bechtel 2008, pp. 22-34). Because a neural function qualifies as "cognitive" once it involves behaviorally relevant information processing, perceptual, affective and social processing mechanisms also fall under this inclusive characterization of cognitive functions (cf. Akagi 2017, p. 7f.). Neuroscientists frequently use sensory stimuli, behavioral or cognitive tasks to study how such information processing mechanisms are executed in response to changes in the environment (Rathkopf 2013). They then characterize entities and activities in these mechanisms as "senders", "channels" and "receivers" that "encode", "transmit" and "decode" information about a stimulus or task. Such terms are not simply mathematical tools to describe the physical properties of neural systems. When applying information processing vocabulary, researchers aim to understand the functional value of neural information processing for the organism (Rieke et al. 1999, pp. 13ff., 54-59, Rathkopf 2017, pp. 325ff.). ${ }^{3}$ The predominant assumption is that information processing contributes to cognitive functions: neural systems process information about the environment to affect the behavior of the organism (deCharms and Zado 2000, pp. 631f., Piccinini and Bahar 2013, p. 456f., Panzeri et al. 2017, p. 471).

In contrast, the sense of "neural function" that is characteristic of noncognitive patches of this concept is expressed well by Marder and Goaillard (2006, p. 563):

Humans, and other long-lived animals, such as turtles and lobsters, have neurons that live and function well for decades. By contrast, ion channel proteins, synaptic receptors and the components of signal transduction pathways are constantly turning over in the membrane and being replaced, with half-lives of minutes, hours, days or weeks. Therefore, each neuron is constantly rebuilding itself from its constituent proteins, using all of the molecular and biochemical machinery of the cell.

When neuroscientists render the meaning of "neural function" this way to search for neural mechanisms, they conceptualize these mechanisms as maintaining or repairing neural or other organismic subsystems in the face of biological turnover (Raichle 2015). They frequently use experimental conditions without sensory stimuli or tasks (e.g. resting state or in vitro conditions) to study how maintenance and repair mechanisms are continuously executed across global states of the organism (e.g. wakeful rest, sleep, anesthesia). The CNS needs to continuously maintain and repair itself because it is an organized system that is not in

\footnotetext{
${ }^{3}$ The historical analyses of the information concept in neuroscience by Garson (2003) and Christen (2006, ch. 3) show that this emphasis on functional value both preceded and remained more important than the purely quantitative and non-semantic notion of “information” from Shannon's information theory.
} 
thermodynamic equilibrium with its environment (Bechtel 2013, p. 7). To uphold its nonequilibrium status, the CNS devotes a lot of energy to maintenance and repair, which enables and constrains information processing (Engl and Atwell 2015, p. 3417f.). Neuroscientists frequently characterize such continuously executed mechanisms as "endogenously active" or "homeostatic" (Marder and Goiallard 2006, Bechtel 2013, Raichle 2015). Such mechanisms are often executed by specialized neural systems such as central pattern generator circuits (sect. 3.1). Hence, they should be conceptualized as noncognitive kinds of "neural function", not just as generic biological functions that all organs execute to maintain homeostasis. Neuroscientists frequently assume that without specialized neural maintenance and repair mechanisms, "the nervous system would be unable to learn, remember, process sensory information, produce movements, or perhaps function at all” (O’Leary et al. 2014, p. 817).

Rather than providing a "mark of cognition"-which is a perennial problem in cognitive science, see Akagi (2017) - I use the graded notion of causal specificity to distinguish between cognitive and noncognitive kinds of neural function. Woodward (2010, p. 307) illustrates the notion of causal specificity in analogy to a radio. The frequency knob has a causally specific effect because changing its value has a distinct effect on the frequency that the radio receives. The on/off button, in contrast, has a causally nonspecific effect because it simply switches all system outputs (e.g., frequency, volume) on or off. Causal specificity is a relational property: the on/off button is nonspecific in relation to its effect on frequency reception. In this paper, I analyze the causal specificity of neural functions in relation to their effect on cognition and behavior. Using Woodward's analogy as an operational criterion, I claim that clear cases of cognitive functions influence cognition and behavior like frequency knobs, whereas clear cases of noncognitive functions work like on/off buttons. They differ in their causal specificity in relation to cognition and behavior. Because causal specificity is a graded notion, there exists a continuum between entities and activities with specific and nonspecific effects on cognition and behavior. In this paper I focus on clear cases that lie at each end of that continuum but elsewhere discuss intermediate cases (see Haueis 2018 and fn. 13).

In the ideal case, causal specificity means that manipulating the input to or any activity in the cognitive mechanism will set the output variable to a value that is distinct from any value that results from other manipulations (Woodward 2010, p. 305; see also Klein 2017). Entities whose activities have causally specific effects are likely to be operative in the cognitive mechanisms that transform a sensory input into a behaviorally relevant output. The notion of "being operative in" is distinct from being necessary or being sufficient (Martin and Deutscher 
1966, pp. 178f.). In the context of cognitive functions, "operative in" refers to the entities and activities that actually bring about the behavioral change when the cognitive system works as it does in the contexts that cognitive neuroscientists care about (see also Craver 2016). Unfortunately, neither Martin and Deutscher nor Craver provide a full-fledged analysis of what it means to be "operative in". In this paper, I take causally specific effects to be evidence for an entity or activities to be operative in a (cognitive) mechanism. I do not claim that such effects define what operative causal conditions are. For example: When visual input arrives in the frontal eye field (FEF), some of its neurons encode a visual saliency map by decoding the retinotopic position with the strongest input signal (Heinzle et al. 2007). Manipulating that activity will change exactly where in the visual field the animal attends its gaze to next. The causally specific effect of FEF activity on gaze behavior is evidence that FEF circuits are operative in the mechanism that brings about changes in eye saccade behavior when organisms attend to their visual environment.

In cases such as the above, information processing correlates with causal specificity because the specific effect of a neural activity will carry a lot of information about the behavioral output of the mechanism. This correlation is weakened if a neural structure contributes to multiple cognitive functions. The structure's activity will be less informative about the output of each individual function. In multifunctional structures, systematicity can serve as additional criterion to attribute behaviorally relevant information processing: there will be a regular and predictable relation between changes in neural activity changes in different cognitive outputs (Klein 2017). ${ }^{4}$ Since multifunctionality is not my main concern here, I focus on causal specificity to distinguish cognitive and noncognitive functions.

Noncognitive patches of "neural function" do not refer to operative conditions with causally specific effects on cognition and behavior. They refer to entities and activities whose maintenance and/or repair functions are necessary enabling conditions for behaviorally relevant information processing. As enabling conditions they likely have causally nonspecific effects on cognitive mechanisms (Woodward 2010, p. 317). The reason for such nonspecific effects is that the CNS must maintain and repair itself and the body constantly while organisms face different cognitive demands when behaving in a changing environment. Many distinct manipulations of the input to or activities in a noncognitive mechanism will have the same coarse-grained effect on cognitive mechanisms. In the simplest case, failure of maintenance and repair switches such

\footnotetext{
${ }^{4}$ I thank Daniel Burnston and an anonymous reviewer for pressing me to clarify this point.
} 
mechanisms "on" or "off". For example: manipulating brain stem circuits that control the breathing rhythm indirectly interferes with attentional eye control because disrupted breathing results in dizziness, confusion, or loss of consciousness. Breathing enables eye control, but brain stem respiratory circuits are not operative in this cognitive function. Conversely, many manipulations of neural gaze control will likely map onto similar, "normal" breathing rhythms because eye movements are not physically taxing. Cognitive effects on breathing are causally nonspecific (Grassman et al. 2016). Because of its nonspecific relation to cognition and behavior, breathing is a clear case of a noncognitive function. The neural entities and activities implementing it enable but are not operative in behaviorally relevant information processing.

Cognitive myopia blinds researchers to noncognitive functions. It prevents them from distinguishing between neural entities that process behaviorally relevant information and those that do not. It also prevents them from acknowledging that there are many signaling functions (e.g., endogenous circuit firing that controls breathing) that do not directly contribute to cognition and behavior. They therefore risk either misattributing information processing to noncognitive mechanisms or discarding them as "noise" or "epiphenomena" when they are in fact necessary enabling conditions for cognitive processing to occur. Philosophers of neuroscience have previously recognized these issues, at least partly. They have argued that researchers can address them by combining experiments that measure or manipulate an entity's while tracking changes in the (cognitive) mechanism with experiments that measure or manipulate the (cognitive) mechanism while tracking changes of an entity's activity (Craver 2007 pp. 139-160). If results from these experiments converge, they provide evidence that an entity or activity is operative in a (cognitive) mechanism, rather than being a background condition that enables it (Silva, Landreth and Bickle 2013, pp. 55-73). A patchwork approach to "neural function" does not compete with these philosophical accounts, but rather adds empirical and conceptual tools that are better suited than "background condition" to describe the relation between cognitive and noncognitive functions (see sect. 4). Backgrounding these enabling conditions prevents researchers from using noncognitive functions to constrain which cognitive models are biologically plausible given the neural infrastructure in which cognitive functions are situated (sect. 3.2). A patchwork approach to "neural function" helps them to avoid these mistakes because it articulates specific conceptual patches to appropriately describe what noncognitive functions are and how they constrain cognitive functions.

I argue that neuroscientists can describe noncognitive functions appropriately by using conceptual patches that dispense with information processing vocabulary. Such patches refer to 
mechanisms that do not represent features of the environment. ${ }^{5}$ For example: FEF activity represents a visual stimulus that is salient to the animal. Neuroscientists mostly use "information" in this narrow sense: sequences of neuronal action potentials carry information about differences in stimuli from the environment (deRieke et al. 1999, deCharms and Zado 2000, Garson 2003, Burnston 2016). Noncognitive mechanisms do not carry information in this narrow sense. For example: because brain stem circuits that control breathing are continuously active, they generate action potentials that carry (almost) no information about differences in stimuli from the environment. Of course, we could give up the narrow sense and allow that many cellular signaling activities besides action potentials representing environmental stimuli carry information (Cao 2014, p. 899). If we apply "information" in this wide sense, we can avoid cognitive myopia in part by acknowledging that many neural signaling functions do not directly contribute to cognition and behavior. Yet, my argument goes beyond the semantics of "information". Even if noncognitive mechanisms carry "information" simply by being responsive to signaling molecules, they are still not operative in cognitive functions. The signals they transform do not bring about the behavioral change that is the output of cognitive functions. A patchwork approach captures this difference by restricting information processing vocabulary to conceptual patches that describe operative conditions for cognition and behavior. To appropriately describe enabling conditions for cognition and behavior, researchers need new patches that describe noncognitive mechanisms as e.g., "endogenously active" or as "maintaining infrastructure". In the next section, I introduce two such conceptual patches that describe subsistence and infrastructural functions.

\section{Noncognitive functions of mesoscale neural circuits}

In this section, I introduce two patches that describe different kinds of noncognitive functions at the mesoscale of neural circuits: subsistence functions implemented by central pattern generator circuits and infrastructural functions implemented by cortical microcircuits. Both functions are noncognitive because they are causally nonspecific enabling conditions for cognition and behavior, and because the respective functional analyses do not require information processing vocabulary. To characterize these noncognitive functions, I follow

\footnotetext{
${ }^{5}$ I restrict this claim to environmental information here because I focus on clear cases of noncognitive functions. Neural structures representing information about the organism itself may present interesting intermediate cases. For example: in the hypothalamus, neural circuits maintain energy homeostasis by representing the energy requirements of the body based on gastrointestinal signals with different time-scales (Beutler et al. 2017).
} 
causal role theories according to which functional analysis involves the decomposition of a containing system $\mathrm{S}$ into causal roles $\phi$ of a system component that contributes to the overall capacity $\psi$ of the system (Cummins 1975, McCaffrey 2015). Adopting causal role theories allows me to show that there are analyses of important neural functions whose target capacities are not cognitive. This sets the stage for the patchwork approach in sect. 4, which shows why the functions picked out by these analyses differ in kind from cognitive circuit functions.

\subsection{Central pattern generators contribute to subsistence functions}

Subsistence functions are necessary to maintain the life of the organism. By "maintaining life" I mean that the organism maintains a homeostatic equilibrium to prevent the deterioration of its parts (e.g., organs and cells). Subsistence fall into two categories: internal systems functions like respiration, digestion and circulation, and external locomotor functions such as swimming, walking, flying and whisking. Internal systems functions are necessary to transform food into inputs for metabolic functions (digestion) and to distribute metabolic products and oxygen throughout brain and body (circulation and respiration). Cognitive processing functions cannot be fueled without the continuous execution of internal systems functions. Internal systems functions enable cognitive processing by maintaining brain and body in homeostatic equilibrium. Likewise, basic locomotor capacities maintain the life of the organism because they enable organisms to execute cognitive functions. Cognitive functions also serve to maintain life, e.g., visual perception helps organisms to find food or avoid predators. But cognitive functions serve to maintain the life of the organism only in virtue of being enabled by subsistence functions, whereas subsistence functions are independent from cognitive ones (see sect. 4).

Subsistence functions are frequently implemented by neural circuits called central pattern generators (CPGs). CPGs can control digestion (lobsters, crabs), circulation (leeches), respiration (rodents, cats, humans) and basic locomotor capacities (all of the above). CPGs can produce rhythmic motor patterns endogenously, i.e. without sensory input (Yuste et al. 2005, Selverston 2010). Recurrent connections, oscillatory membrane properties and neuromodulatory input allow them produce motor rhythms even if parts of the organism are damaged or absent. The functional analysis of CPG circuits can be schematized as follows: 


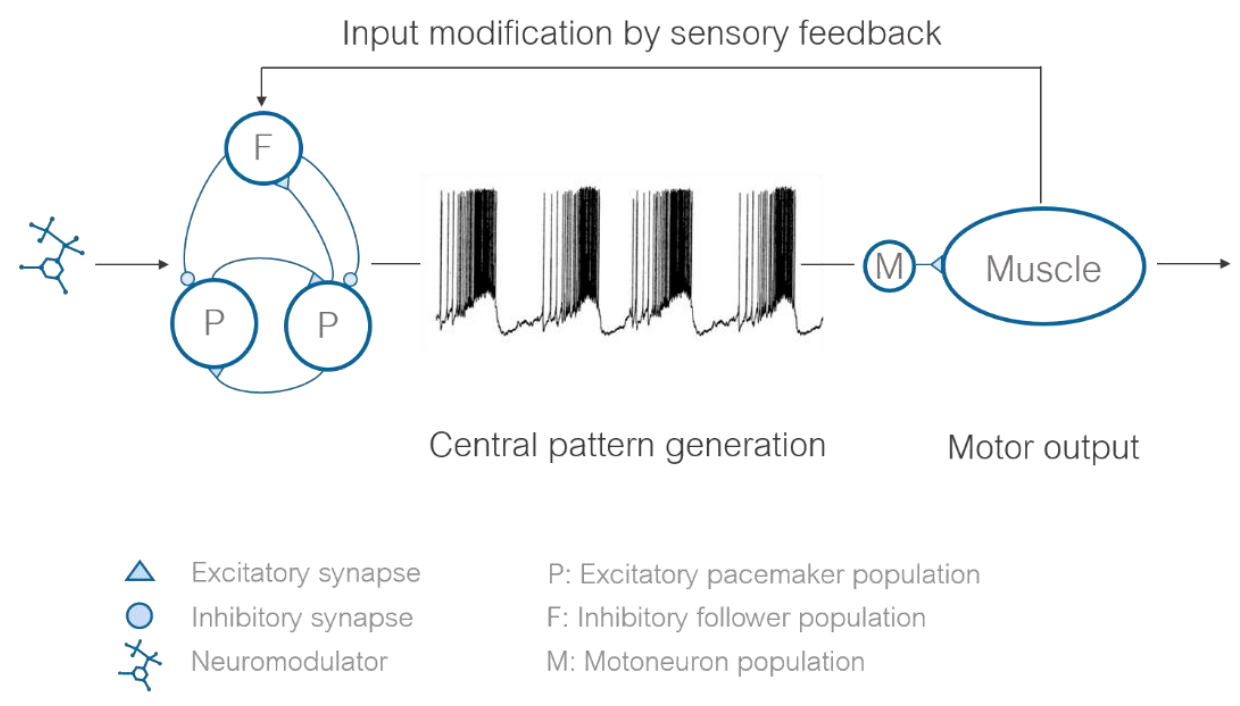

Fig. 1: Schematized functional analysis of CPG circuits.

Figure 1 depicts how researchers decompose a motor system's capacity for rhythmic movement $(\psi)$ into the role of CPGs to endogenously generate the motor rhythm $\left(\phi_{C P G 1}\right)$ and the role of motoneurons to forward $\left(\phi_{C P G 2}\right)$ this rhythm to the muscle, which moves $\left(\phi_{C P G 3}\right)$ the motor system in that rhythm. The crucial point is that endogenous rhythm generation $\left(\phi_{C P G I}\right)$ does not depend on sensory inputs. In the CPG architecture depicted above, two pacemaker populations recurrently excite each other and a follower population, which in turn inhibits the pacemakers. These circuit interactions create an oscillatory rhythm as output. CPG circuits can create such rhythms when isolated from the organism, and sometimes even without neuromodulatory input (Marder and Bucher 2007). ${ }^{6}$ In the intact organism, however, the containing system for functional analysis is the motor system to which the CPG rhythm contributes.

Neural functions of this kind are noncognitive because they are causally nonspecific enabling conditions for cognition and behavior. Consider first internal systems CPGs. I already discussed breathing as an exemplary noncognitive function above. Digestion and circulation work in the same way. If CPGs controlling these functions are necessary for maintaining life, they are also necessary for cognitive processing. But it would be wrong to say that respiratory, circulatory or digestive CPG activities are operative in any particular cognitive processing mechanism they enable. Failure of digestive, circulatory or respiratory activity will indirectly interfere with many such mechanisms because it will cause metabolic dysfunctions and shortage of energy supply. Such indirect interferences are causally nonspecific either because they shut

\footnotetext{
${ }^{6}$ Neuromodulators are signaling molecules that influence the conductance properties of many neurons over long periods of time. In the intact animal, some combination of neuromodulators is necessary to initiate CPG rhythm generation (Marder 2012).
} 
cognitive mechanisms “off”, or because they perturb many cognitive mechanisms at once (cf. Craver 2007, pp. 148f.). Because internal systems CPGs have such causally nonspecific effects, they are not operative in, but enabling conditions for cognitive functions.

Because the CPG concept describes circuit activity that contributes to motor systems, it has "the advantage of bypassing the question of how sensory information is coded by single neurons or populations of neurons" (Selverston 2010, p. 2331). Because researchers do not have to answer the question how CPGs encode sensory information from the environment, information processing vocabulary is not required to understand what these circuits do. Neuromodulatory inputs do not need to be characterized as senders of information from the environment because they are produced internally by neurosecretory structures to keep CPG circuits continuously active (Marder 2012, p. 2). CPG units firing action potentials also need not be characterized as processing information about the environment because they form a recurrent circuit that keeps itself active independently of sensory inputs (cf. Cao 2011, p. 11). Describing CPG functions in terms of narrow information processing would be inappropriate because the underlying circuits do not encode sensory inputs to generate motor outputs. What researchers need to describe instead is how membrane properties, synaptic connectivity and neuromodulators endow a CPG circuit with endogenous rhythm generation $\left(\phi_{C P G 1}\right)$, and how this rhythm drives motoneurons $\left(\phi_{C P G 2}\right)$ and moves muscle groups $\left(\phi_{C P G 3}\right)$. Such circuits perform noncognitive functions. Their component cells do not encode sensory information, and they can generate rhythms in the absence of peripheral or cortical feedback.

Although researchers do not require information processing vocabulary for functional CPG decomposition, internal subsistence functions are not causally disconnected from sensory and cognitive processing systems. Behavioral and cognitive states like physical exercise or emotional arousal modify the rate of cardiac and respiratory rhythms (Appelhans and Luecken 2006, Homma and Masaoka 2008, Luque-Casado et al. 2013). I call such feedback effects input modification (Fig., 1 arrow, top) because sensory input is not necessary for, and has nonspecific effects on endogenous rhythm generation. A recent meta-analysis, for instance, revealed that increases in cognitive load correlate with higher breathing rates (Grassmann et al. 2016). But this effect occurred regardless of whether the load of a memory, attention or mental arithmetic task was manipulated. Because of input modification, endogenous rhythm generation is consistent with the fact that quantitative details of the rhythm (e.g., frequency or amplitude) can be changed by sensory or cortical feedback. The conceptual patch that describes noncognitive 
subsistence functions allows researchers to separate input modification from the necessity of maintaining internal systems capacities at all times.

So far, I focused on the decomposition of internal subsistence functions. I now introduce the whisking CPG in the rat subcortex as an example of a noncognitive circuit that implements an external locomotor function. Whisking is the rhythmic movement of facial hair by which rodents identify food, navigate their environment and interact with mates. The whisking CPG example serves a dual purpose. First, it shows that cognitive myopia is not only inappropriate when researchers study neural systems with no obvious cognitive function (e.g., the digestive system in invertebrates). It is also inappropriate when researchers study neural systems with cognitive functions (here: the tactile system in rodents). Such systems can still implement noncognitive functions at the same scale (here: the mesoscale of circuits) at which cognitive ones are implemented. It would be inappropriate to describe these noncognitive, enabling functions as "processing information" or as being "operative in" cognition. Second, the whisking CPG example shows why researchers need both cognitive and noncognitive concepts to examine the relation between decomposed functions. They need both subsistence and information processing concepts to recompose the whisker system by situating it in a larger containing system (cf. Bechtel and Abrahamsen 2009).

In rodents, the capacity of whisking is an enabling condition for various cognitive functions. If whisking fails, rodents become unable to find food or avoid prey (navigation/object recognition) or to identify mating partners (social touch). Thus, whisking is a subsistence function that is necessary for rodents to stay alive. After earlier ablation experiments revealed that whisking occurs without sensory or cortical feedback, Cramer et al. (2007) hypothesized that a subcortical CPG circuit produces the whisking rhythm. They predicted that serotonin modulates vibrissa motoneurons in the facial nucleus via a persistent inward current that depolarizes the neurons until rhythmic firing occurs. When the researchers first applied a serotonin type 2 agonist and then inhibitory agonist riluzole to brain stem slices, vibrissa motoneurons started and then stopped firing at whisking frequencies $(2-17 \mathrm{~Hz})$. From these in vitro experiments, they concluded that serotonin is both necessary and sufficient to generate the whisking rhythm. They complemented these results with in vivo experiments which suggested that premotoneurons in the pargigantocellular nucleus (PGN) supply endogenous serotonin input. Because this nucleus receives connections from the vibrissa motor cortex (VMC), they microstimulated the VMC while measuring PGN neurons and intrinsic vibrissa muscle responses. Serotonergic PGN spiking and whisking frequency were positively correlated. 
Therefore Cramer et al. applied the CPG concept to this system: the vibrissa motoneurons transform a tonic serotonergic drive into the whisking rhythm. Because the tonic input in the whisker system is endogenously produced serotonin, Cramer et al.'s experiments do not refer to transient sensory input. Their functional analysis does not invoke information processing vocabulary. It does not explain rhythmic whisking as the outcome of neural processing of information about the environment.

Ebbesen et al. (2017) furthermore suggest that information processing in the VMC and other cortical areas has a causally nonspecific effect on the subcortical whisking CPG. They recorded VMC responses during exploratory whisking, object touch and social touch. In all three tasks, over $80 \%$ of the modulated neurons decreased firing compared to the baseline condition of wakeful rest. VMC firing seems to neither have a task-specific effect, nor to activate whisking. When stimulating the VMC below spiking threshold during social touch, the researchers recorded increased hyperpolarization in layer 5 output cells. Above-threshold stimulation and pharmacological blockage of VMC firing caused the rats to protract their contralateral whiskers and shortened social whisker touches. These results "support a model in which VMC activity supresses whisker behavior, perhaps by gating a downstream whisking central pattern generator" (ibid., p. 87). In this model, the VMC inhibits a continuously active CPG circuit in a causally nonspecific manner, rather than episodically activating a silent subcortical nucleus on task demand. VMC activity and sensory processing (e.g., in barrel cortex) supresses this rhythm equally across all contexts in which whisking is ecologically irrelevant. This circuit organization can be depicted as follows:

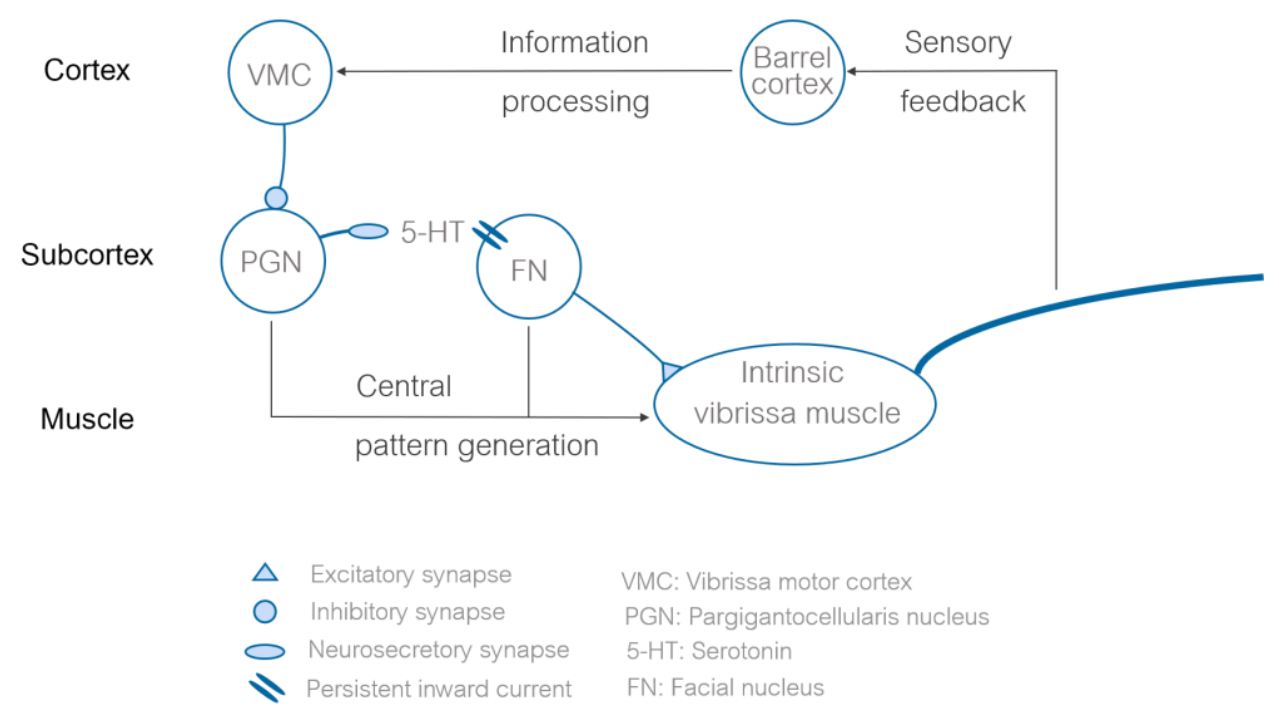

Fig. 2: Simplified circuit diagram for whisking generation in rats. 
Figure 2 shows that the whisking rhythm can be centrally generated (black arrows, lower left), independently of sensory feedback and cortical information processing (black arrows, upper right and middle). The flow of behaviorally relevant information does not encompass the subcortical CPG circuit itself, because that circuit is continuously active as long as the PGN endogenously generates serotonin. When the behaving animal responds to transient environmental inputs, CPG activity is subject to input modification via the presumed inhibitory connection between VMC and PGN neurons. The diagram therefore exemplifies how a noncognitive functional analysis divorces endogenous rhythm generation from narrow information processing in neural systems underlying overt behavior such as whisking. It thus challenges Bechtel's analysis of endogenous brain activity (e.g., whisking CPG activity) as being operative in cognitive information processing because it "directly affects mental processes and behavioral activities" $(2013,19)$.

The above diagram shows how circuit researchers can avoid cognitive myopia by recognizing that the rodent brain executes both cognitive and noncognitive functions. The diagram can be used to recompose subsistence and information processing functions in a larger containing system (Bechtel and Abrahamsen 2009). For example: Moore et al. (2013) discovered that parts of the reticular formation also belong to the whisking CPG, because chemically activating them drives vibrissa motoneurons while lesioning them inhibits ipsilateral whisking. Since whisking unit firing was coupled to the breathing rhythm, the authors suggest that the brainstem respiratory CPG could act as a "master clock" for facial behaviors (e.g., whisking, chewing, licking). These findings add further details to the pathway of central pattern generation in Fig. 2. Conversely, Sreenivasan et al. (2014) used viral vectors and optogenetic stimulation to visualize and manipulate parallel somatosensory and motor pathways that selectively activate or supress contralateral whisker movements. These findings add further details to the pathway of cortical information processing. Adding the subsistence function of breathing or the cognitive function of somatosensory processing to the diagram shows how researchers can recompose noncognitive and cognitive functions by specifying their interactions in a larger containing system.

To summarize: CPGs with subsistence functions are necessary to maintain the life of the organism and enable various cognitive functions to occur. Endogenous rhythm generation makes subsistence functions independent from sensory and cortical feedback. Cognitive myopia tempts researchers to interpret this endogenous activity as cognitive (Yuste et al. 2005, Bechtel 2013). But CPGs are not operative in the cognitive mechanisms from which they 
receive input modification. To avoid shoehorning them into cognitive vocabulary, CPGs should be analysed without information processing concepts or cognitive tasks. The conceptual patch of "subsistence function" allows researchers to appropriately understand what these circuits do, whereas a cognitively myopic picture of "neural function" does not.

\subsection{Cortical microcircuits contribute to infrastructural functions}

Infrastructural functions prevent neural system damage while behaviorally relevant information processing occurs. They occur "irrespectively of the particular systems-level function to which any of the systems happens to be dedicated" (Merker 2013, p. 402). They are noncognitive because they are causally nonspecific enabling conditions for cognition: if they fail, circuit infrastructure gets damaged and becomes unable to process information. Because cognitively myopic researchers tend to overlook infrastructural functions, they fail to explain how cognitive functions are constrained by the physiological apparatus that maintains the brain's structural integrity. Below I focus on the capacity of gain control to argue that infrastructural functions provide crucial constraints on cognitive functions in cortical circuits.

The neuroscientific concept of gain control originated from exploratory experiments by Kirschfeld (1992), who found that the analogues of cortical gamma oscillations in the fly optic lobe do not systematically depend on visual stimulus parameters. Unlike Ghose and Freeman (1992) who concluded from similar results that oscillations in the cat visual cortex are epiphenomenal, Kirschfeld freed himself from cognitive myopia. He sketched the noncognitive mechanism of gain control in which oscillations provide a negative feedback signal to activate an inhibitory brake (1992, p. 4767). ${ }^{7}$ Because oscillations arise from excitatory-inhibitory interactions, they signal that further activation leads to circuit over-excitation. To prevent overexcitation, the inhibitory brake controls the gain of excitatory circuit elements by decreasing their spiking probability. Without gain control preventing over-excitation, neural infrastructure gets damaged and information processing cannot occur.

Kirschfeld's mechanism sketch can be applied to cortical circuits by adding explanatory details to the "canonical microcircuit" (CMC). The CMC describes two recurrently connected excitatory populations whose activity is balanced by a recurrently connected inhibitory

\footnotetext{
${ }^{7}$ Sometimes the term "gain control" appears in explanations of how neurons adapt their responses to variations in sensory input (e.g. contrast or luminance in the visual system, cf. Heeger 1992). As will become clear below, this multiplicative effect to enhance narrow information processing is better described as neural gain modulation, whereas gain control refers to the divisive and switch-like effect to prevent circuit damage.
} 
population (Douglas and Martin 1991). Researchers can use the CMC concept to analyse both cognitive and noncognitive functions of cortical circuits. Consider first a cognitive analysis using the CMC concept:

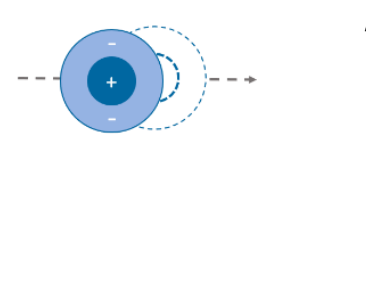

Thalamic input in A precedes input in $B$
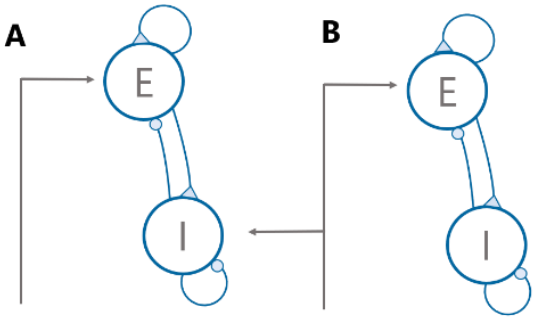

Preferred direction

E: excitatory CMC population I: inhibitory CMC population

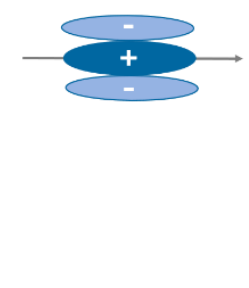

Direction-selective cortical output

Fig. 3: Direction-selective circuit responses in primary visual cortex (V1). Adapted from Douglas and Martin (1991, Fig. 5).

Figure 3 exemplifies how researchers analyse mesoscale circuits when working on a cognitive patch of "neural function". Direction-selective responses are explained by the temporal difference in non-selective input from the visual thalamus (blue circles, left) to two simplified CMC modules $(A$ and $B$ ). When thalamic activity is temporally displaced in the preferred direction (dashed grey arrow), module $A$ recurrently excites itself and module $B$, leading to a direction-selective output. When thalamic activity occurs in the non-preferred direction, inhibitory neurons in module $A$ activate first and prevent recurrent excitation of $A$ and $B$. Such cognitive functional analyses add explanatory details about behaviorally relevant information processing to the $\mathrm{CMC}$ model. By contrast, noncognitive functional analyses add explanatory details about infrastructural maintenance to the $\mathrm{CMC}$ model. In gain control, these details specify the causal role of negative oscillatory feedback $\left(\phi_{G C 1}\right)$ and the inhibitory brake $\left(\phi_{G C 2}\right)$.

First, cortical gamma oscillations $(30-80 \mathrm{~Hz})$ can provide a negative feedback signal for gain control $\left(\phi_{G C l}\right)$. Excitatory cell firing causes inhibitory cells to fire, which in turn inhibit the excitatory cells. This interaction creates an oscillatory wave with excitatory spikes occurring during the peak and inhibitory spikes occurring during the through of the wave (Bartos et al. 2007). Because they arise from circuit interactions, gamma oscillations can signal how active a given circuit is. This negative feedback role $\left(\phi_{G C l}\right)$ can occur irrespective of the particular information processing task the circuit contributes to. When analysing gain control, researchers can add $\left(\phi_{G C l}\right)$ to the $\mathrm{CMC}$ model, because it refers to circuits that oscillate in the gamma range (Grillner et al. 2005). Second, chandelier cells can act as inhibitory brakes in the cortex $\left(\phi_{G C 2}\right)$. 
They exclusively synapse onto the axon hillocks of excitatory pyramidal neurons. Because action potentials are generated at the axon hillock, chandelier cells can directly prevent pyramidal firing. When Zhu et al. (2004) disrupted the excitation-inhibition balance in barrel cortex circuits, chandelier firing increased 22 fold, whereas other neurons increased firing only 1.6fold. They concluded that "chandelier cells may function as the last defense to keep network excitability from going out of control" (ibid., p. 5107). When analysing gain control, researchers can add $\left(\phi_{G C 2}\right)$ to the $\mathrm{CMC}$ model by separating the infrastructural role of chandelier neurons (prevent over-excitation) from the infrastructural role of other inhibitory neurons (maintain excitation-inhibition balance). Besides adding $\left(\phi_{G C l}\right)$ and $\left(\phi_{G C 2}\right)$ to the $\mathrm{CMC}$, the gain control analysis specifies switch points for this infrastructural function. At low pyramidal firing rates, no gain control occurs because chandelier cells provide a "window of excitation": their hyperpolarizing effect decays faster than the depolarizing effect (Woodruff et al. 2010, Fig. 2c). At intermediate firing rates, gain control sets in because chandelier inhibition outruns the initial depolarizing effect (Merker 2013). At high pyramidal firing rates, chandelier inhibition increases drastically to prevent over-excitation (Zhu et al. 2004).

The functional analysis of gain control can now be formulated as follows (see Fig. 4). When cortical microcircuits start firing in response to sensory or cortical inputs, chandelier cells enable information processing because they provide pyramidal cells with a "window of excitation". Over time, information processing leads to excitatory-inhibitory interactions, generating gamma oscillations within the circuit. If these oscillations indicate intermediate circuit firing, chandelier inhibition results in divisive gain control: pyramidal firing rates are reduced by a divisive factor (Abbot and Chance 2005, p. 148). When the pyramidal cells continue to increase their firing, however, chandelier cells act as inhibitory brakes by subtractively decreasing pyramidal firing. The role of gamma oscillations in gain control would be "analogous to the "ringing" that can be used to tune a microphone - amplifier - loudspeaker system to keep it from breaking into howling feedback" (Merker 2013, p. 406). This comparison fits well with the observation that gamma oscillations are transitioning into seizure activity in in vitro experiments when inhibition is missing, and in patients with photosensitive epilepsy where chandelier cells are scarce or absent (de Felipe 1999).

The infrastructural function of gain control is noncognitive because negative gamma feedback and chandelier activities are causally nonspecific enabling conditions for cognitive functions. Consider first gamma oscillations. A puzzling finding is that they occur together with some, but not other visual stimuli (Ghose and Freeman 1992, Hermes et al. 2015). The gain 
control analysis explains this irregularity as follows: what influences gamma oscillations is not which stimulus feature is processed, but how much circuit activity that processing requires. When experimenters manipulate a cognitive function by presenting a certain stimulus, they often observe increased circuit firing. But whether this increase is enough to induce oscillations does not depend on the type of behavior to which the cognitive circuit function contributes. Cognitive manipulations have nonspecific effects on oscillations because they are mapped to circuit activity levels relevant for gain control. Gamma oscillations do not provide negative feedback about the content of information processing, but about the activity level that a circuit requires to process information. Consider now chandelier cells. Any effect of information processing is mapped onto one of three states: (i) window of excitation, (ii) divisive gain control, (iii) inhibitory brake. That mapping does not depend on the content of the information processing task. It depends on physiological circuit parameters, like firing rate, axon hillock polarization, and negative gamma feedback. These parameters do not specify informational content, but causal switch points for gain control to prevent damage of neural infrastructure.

This lack of causal specificity is evidence that infrastructural functions like gain control are enabling conditions for cognitive functions. Properly working infrastructural functions enable many behaviors controlled by cortical circuit processing (e.g., motion detection, eye gaze control). But if they are disrupted, cognitive mechanisms controlling these behaviors are "shut off". When chandelier cells are lost, sensory input causes seizure activity instead of regular information processing. Gain control is thus a fundamental constraint on cognitive functioning: if it does not prevent damage, the CNS cannot process the sensory information it needs to adaptively respond to the environment. The specific composition of infrastructural functions matters to explain how exactly information processing can work. Reference to specialized gain control units - chandelier cells - is required to explain exactly how circuits in the cortex can process information at all. The conceptual patch describing infrastructural functions allows researchers to recognize these constraints, whereas a cognitively myopic view on cortical circuit function does not. Researchers working on this patch do not require informational concepts because they can use physical, non-semantic properties to describe the signaling roles of entities or activities that implement infrastructural functions (cf. Cao 2011, p. 6). Negative gamma feedback indicates that processing occurs at a particular circuit activity level, but not which kind of information is being processed (Merker 2013). Chandelier cells do not process sensory information but monitor circuit activity levels to act as divisive gain 
controllers or inhibitory brakes (Zhu et al. 2004). Consequently, the relation between gain control and information processing can be visualized as follows:

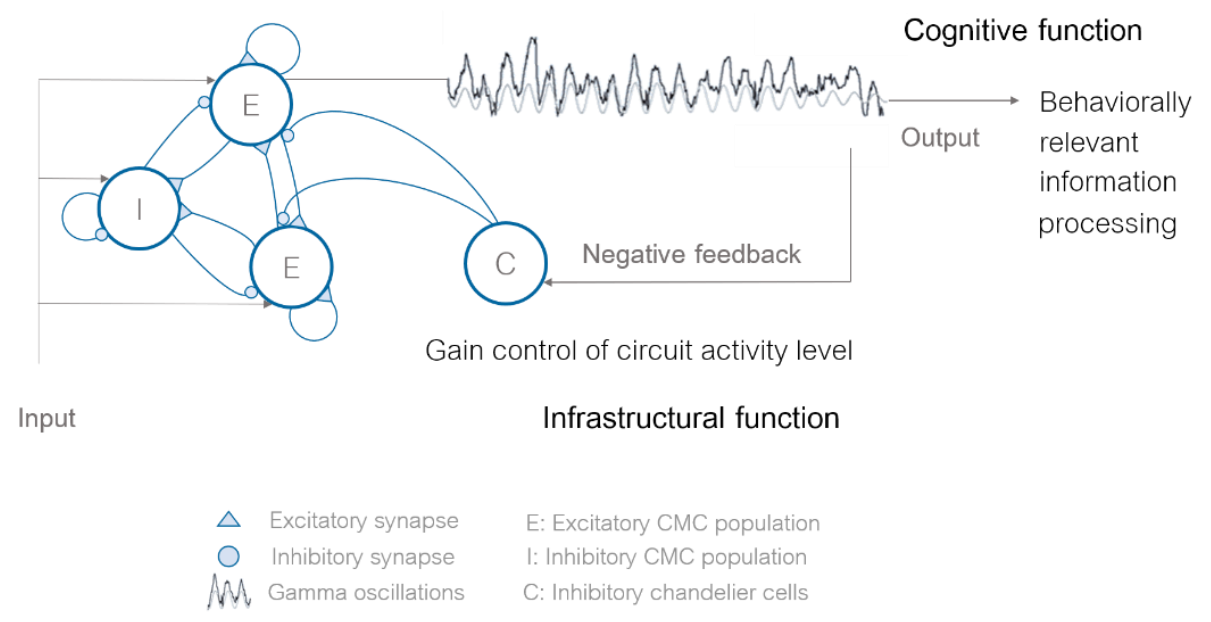

Fig. 4: Information processing and gain control in cortical microcircuits.

The above diagram shows that we can overcome cognitive myopia by recognizing that cortical microcircuits execute both cognitive and noncognitive functions. To analyse these functions, researchers can use the $\mathrm{CMC}$ concept (E and I nodes and connections, left). When studying cognitive functions, they add explanatory details about the input signal (stimulus type), signal transformation (extraction of behaviorally relevant features), and the receiver of the signal output. Cognitive functional analyses (arrows from lower left to upper right) refer to circuit elements that are operative in behaviorally relevant information processing (e.g., computing stimulus direction or controlling eye saccade behavior). When studying noncognitive functions like gain control, researchers add explanatory information about circuit activity levels, negative feedback and divisive or subtractive inhibition. Infrastructural analyses (arrow, lower right) therefore refer to circuit elements that enable cognitive circuit activity by preventing damage.

Infrastructural functions do not only enable, but also constrain cognitive functions. To incorporate these constraints, researchers need to recompose infrastructural and cognitive functions. Consider multiplicative gain modulation, which increases pyramidal firing rates by a multiplying factor. Ni et al. (2016) studied this effect in macaques to test the "communication through coherence hypothesis": stimulus-dependent gamma oscillations in V1 entrain V4 neurons, which temporarily enhances information transmission. During an attention task, V4 activity oscillated in the gamma frequency $(40-60 \mathrm{~Hz})$. The multiplicative gain of the oscillatory activity was behaviorally relevant because macaques shifted their attention quickest when the strongest gamma-phase-locked firing occurred. Infrastructural gain control constrains this 
cognitive function as follows: when chandelier cells depolarize the axon hillock, they provide a "window of excitation" during which multiplicative gain modulation can occur. When pyramidal firing continues, increased chandelier inhibition levels replace multiplicative gain modulation with divisive gain control. This recomposition assigns a positive feedforward role to gamma oscillations as enhancing information transmission between two areas (in Ni et al.'s case: V1 and V4), and a negative feedback role to oscillations for controlling circuit gain within an area. The constraint in this recomposition is that gamma oscillations can only enhance information transmission below the switch point for divisive gain control. Researchers must not ignore such infrastructural functions if they want to explain how cognition is coherent with maintaining the brain's structural integrity. ${ }^{8}$

In this section, I introduced two conceptual patches that appropriately describe noncognitive circuit functions. Such noncognitive functions enable cognition and maintain the structural integrity of the CNS and other parts of the body. Cognitive myopia prevents researchers from adequately studying these functions because it suggests that "neural function" univocally refers to cognitive information processing mechanisms. In the next section, I articulate a semantic picture of "neural function" that overcomes cognitive myopia by recognizing that this concept has both cognitive and noncognitive patches of local application.

\section{A patchwork approach to "neural function" at the mesoscale}

The examples from the previous section suggest that neuroscientists use the concept "neural function" to refer not only to cognitive, but also to noncognitive kinds of function. Because cognitive myopia only recognizes applications of "neural function" to cognitive functions, it provides an erroneous semantic picture of this concept. In this section, I propose that a patchwork approach to "neural function" is a proper corrective to cognitive myopia. So far, I followed causal role theories of function to describe how researchers analyse subsistence and infrastructural functions. Causal role theories can acknowledge that neural structures contribute to capacities that are not cognitive, such as breathing or gain control. But they do not provide resources to show why these neural functions differ in kind from functions that contribute to cognitive capacities. Absent further mechanistic or evolutionary constraints (Craver 2007, ch. 4, Šustar 2007), causal role theories only support the claim that different functional analyses in

\footnotetext{
${ }^{8}$ Further constraints can be added by including other infrastructural functions such as the role of microglia cells to remove amyloid plaques when stimulated in the gamma frequency (Iaccarino et al. 2016). Plaque removal enables and constrains learning and memory mechanisms because it prevents onset of neurodegenerative diseases.
} 
neuroscience pick out different capacities with different vocabulary. Hence one cannot block the argument that such differences in using "neural function" are merely superficial, and that a unified conceptual framework to analyse all neural functions remains preferable. Cognitive myopia suggests that cognitive and information processing concepts provide such a framework. To undercut its plausibility, one needs to show that a) cognitive, subsistence and infrastructural functions differ in kind and that b) when analysing such functions, neuroscientists render the meaning of "neural function" in different ways. A bare bones causal role theory provides no resources to support a), and therefore cannot be used to infer that b) is the case. ${ }^{9}$ In contrast, a patchwork approach supports both claims because it shows exactly why conceptual patches of "neural function" distribute themselves over various kinds of function at the mesoscale.

To understand why conceptual patches refer to different kinds, consider the concept of "hardness" in materials science (Wilson 2006). When applying "hardness" to metals, researchers use indenter tests to measure the physical property of yield strength. When applied to elastomers such as rubbers, however, indenter tests supply anomalous results because the elastic surface of rubbers recovers too quickly after indentation. Researchers therefore use durometer tests in elastomers, which refer to Young's modulus of elasticity. These local applications plus experimental methods form two patches in the patchwork structure "hardness" (ibid., pp. 335-45). Yet the indenter/yield strength and durometer/elasticity patches partially overlap, because they both supply sensible results for polyamide materials like nylon. Scientists can therefore use the two patches of "hardness" to describe three kinds of material: metals, polyamides and elastomers. The patchwork structure of "hardness" arises as "a consequence of members of certain higher-level kinds [...] to exhibit similar behaviors - that metals are all susceptible to [indenter] tests is both a fact about how the concept 'hardness' behaves around that kind of materials, as well as a fact about the class of materials themselves" (Bursten 2016, p. 7f.). Therefore, the patchwork structure of a concept like "hard" is not simply determined by the tests used to measure it; it crucially depends on how empirical properties (here: yield strength, elasticity) are distributed among the kinds of entities that the concept picks out.

Patchwork approaches claim that the heterogeneous structure of concepts is useful to scientists because it tracks the distribution of kinds in their application domain. I use "kind" here in a methodological, not in a semantic or metaphysical sense. As methodological tools, kind concepts enable scientists to perform experimental interventions, confirm hypotheses and

\footnotetext{
${ }^{9}$ I thank an anonymous reviewer on pressing me to clarify why causal role theories are insufficient to overcome cognitive myopia.
} 
construct explanations (cf. Bursten 2016, p. 5). Methodological analyses in philosophy of science do not aim to show how kind concepts carve nature at its joints or possess a fixed reference. They rather aim to show how concepts pick out properties that are interesting to scientists because they can be "robustly detected, measured, manipulated, and intervened upon" (ibid., p. 8). Patchwork approaches show that concepts can fulfil such roles because scientists introduce experimental conditions that provide - at least preliminary - application criteria of the concepts to entities or activities in the domain of inquiry (Haueis 2014, 2018). Such local experimental conditions can operationally define new concepts without being supported by a unified theory about the domain (cf. Wilson 2006, pp. 180ff.). Rather than being determined beforehand, the application domain of such novel concepts is discovered in the process of extending them to novel cases in a stepwise fashion. ${ }^{10}$ If such stepwise extensions shift the reference to entities with novel properties or behaviors in the domain, the concepts will develop partially overlapping local patches of application. Ideally, each patch picks out a different kind of entity, with intermediate cases lying in the overlapping region of two patches. A conceptual patch provides researchers the experimental and conceptual tools (e.g. instruments, operational definitions, inductive inference rules) to detect, measure, manipulate or predict the properties or behaviors of that kind. Conceptual patchwork structures as a whole provide researchers with the methodological tools to classify kinds of entities and activities in their domain of inquiry.

By adopting a patchwork approach to "neural function", I propose that this concept possesses a heterogenous structure of locally adapted applications. Such a structure is useful to neuroscientists because it tracks the distribution of different kinds of function in the neural domain. At the mesoscale of neural circuits, "neural function" consists of three patches that refer to subsistence, infrastructural and cognitive functions. Cognitive myopia mistakenly suggests that this concept only tracks one kind of circuit function-behaviorally relevant information processing. In contrast, a patchwork approach captures the fact that neuroscientists use "neural function" to refer to (at least) two other kinds of circuit function that are not appropriately characterized as being cognitive. A patchwork approach recognizes the differences in cognitive and noncognitive functional analyses as part of the empirical and conceptual tools to track different kinds of circuit function. When neuroscientists use these tools to study neural circuits under different experimental conditions, they render the meaning of

\footnotetext{
10 Patchwork approaches thus differ from holist theories which posit a unified theory or essentialist theories that posit a universal referential relation to a natural kind to explain how concepts acquire their meaning. Patchwork approaches are better suited to analyse how concepts behave in scientific practice, where exploratory concept formation and changes of reference occur frequently (see Haueis 2018, chs. 2 and 3 for historical examples).
} 
"neural function" in different ways. For example: when electrophysiologists track how manipulations of sensory input change the circuit output in specific ways, they render the meaning of "neural function" cognitively. They use this concept to refer to operative conditions in behaviorally relevant information processing. Cognitive myopia suggests that such a cognitive rendering works for all instances of "neural function". In contrast, a patchwork approach shows why the cognitive patch of "neural function" does not provide the right tools to robustly detect, manipulate and predict the behavior of circuits with noncognitive functions. For example: varying sensory inputs to detect changes in circuit output is an inappropriate method to understand what CPG circuits do. Instead, CPG researchers track how endogenous rhythm generation remains invariant under in vitro and in vivo conditions to support the view that sensory and cortical inputs are not necessary to execute functions that maintain the life of the organism (Marder and Bucher 2007). Conversely, searching for circuit activity that is invariant under in vivo and in vitro conditions supplies unreliable results when studying cognitive circuit functions (e.g. in vitro results may be unreliable to predict cognitive activity if that activity changes with behavioral context, cf. Burnston 2016). The reason why the applicability of these methods is confined to local patches is that they are tailored to detect, measure and manipulate different kinds of neural circuit function.

In the case of "hardness", the form of the patchwork structure depends on how empirical properties like yield strength or Young's modulus of elasticity are distributed among kinds of materials (e.g., metal, polyamides, elastomers). Shifts between patches of "hard" are marked by changes in these properties. What empirical properties distinguish kinds of functions as the basis of different conceptual patches of "neural function"? As indicated above, I think that causal roles and capacities are insufficient: the fact that circuits can contribute to cognitive, subsistence or infrastructural capacities does not show that the underlying circuit functions are different in kind. The property of causal specificity is also insufficient: it only distinguishes cognitive circuit functions with causally specific effects from those with nonspecific effects. But it does not distinguish subsistence from infrastructural functions. To articulate a patchwork structure that distinguishes all three circuit functions, I therefore add two relational properties of neural circuits: their mechanistic organization and their evolutionary relations. ${ }^{11}$ Shifts in mechanistic organization and evolutionary relations mark shifts between the cognitive, subsistence and infrastructural patches of "neural function".

\footnotetext{
${ }^{11}$ Both properties have been suggested as additions to a bare bones causal role theory of neural function (Garson 2011, McCaffrey 2015). I aim to use both as constraints on the patchwork structure of "neural function", since each may be useful for functional analysis in different research contexts.
} 
To understand how circuits with subsistence functions are mechanistically organized, recall the whisking system (Fig.3). In that system, serotonin is necessary and sufficient to cause rhythmic firing. Therefore, whisking CPGs can endogenously create rhythms without sensory input or cortical feedback. I call this type of circuit organization mechanistic decoupling from cognitive functions. Invariance of rhythmic CPG activity under in vitro and in vivo conditions counts as evidence for the mechanistic decoupling of subsistence from cognitive functions. To paraphrase Bursten (2016): that all CPGs with subsistence functions are susceptible to mechanistic decoupling is both a fact about how the concept "neural function" behaves around that kind of circuit, as well as a fact about the class of circuits themselves. In contrast, infrastructural functions are essentially coupled to cognitive information processing mechanisms. Gamma oscillations and chandelier inhibition occur in many cognitive contexts because the respective behavioral tasks places increased functional demands on cortical microcircuits. Without increased functional demand, the gain control mechanism is not executed. Negative gamma feedback may therefore be experimentally inseparable from the information processing mechanisms with which it co-occurs (cf. Merker 2013, p. 409). Yet, infrastructural functions are not part of the flow of behaviorally relevant information (Fig. 4).

To understand how the three mesoscale functions are related evolutionarily, recall first that I argued that CPG circuits do not process behaviorally relevant information, but maintain the life of the organism. That means that if circuits with cognitive functions evolved from CPGs, then CPGs which were previously adapted for subsistence functions subsequently acquired novel, cognitive processing functions. This evolutionary relation between two functions is called exaptation (Gould and Vrba 1982). In contrast, the evolutionary shift from subsistence to infrastructural functions does not involve a novel function, but only a shift in the containing system to which they contribute. Whereas subsistence functions contribute to systems outside the CNS (e.g., lungs), which in turn maintain the entire organism, infrastructural functions contribute to maintaining (a part of) the CNS itself. That means that if infrastructural functions evolved from CPGs with subsistence functions, then CPGs became internalized to maintain neural infrastructure. In evolutionary neuroscience, this process is called "encephalization of motor rhythms" (Yuste et al. 2005, 477, see also below). By adding the properties of decoupling, coupling, exaptation and encephalization and the circuit diagrams from section 3 we can now visualize the patchwork structure of "neural function" at the mesoscale as follows: 


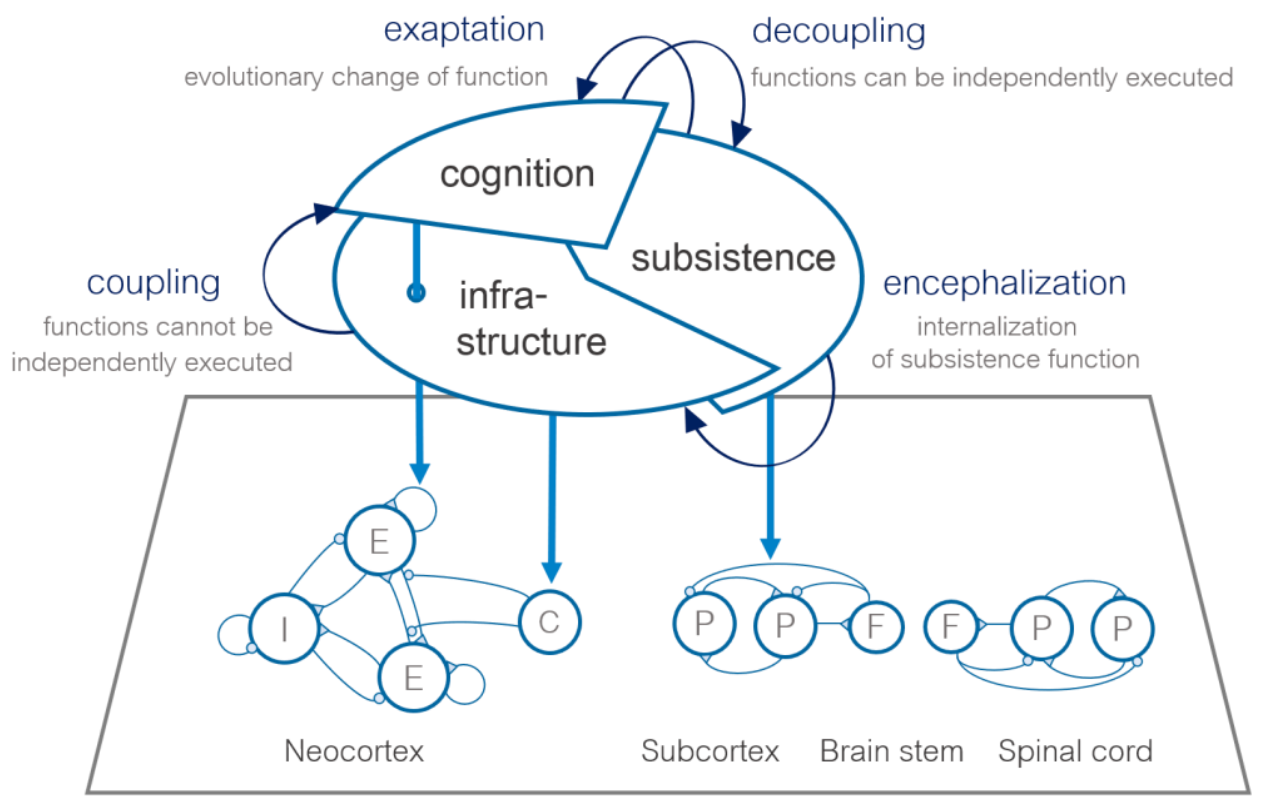

Fig. 5: A patchwork approach to "neural function" at the mesoscale.

The above diagram can help us move beyond cognitive myopia because it reveals the actual material inferential structure of cognitive and noncognitive applications of "neural function" in mesoscale circuit research. These local applications are evaluated according to the functional patch that researchers work on (pitch circles). In the spinal cord, brain stem, and parts of the subcortex, researchers work on the subsistence patch because they study CPG circuits that are necessary to maintain the life of the organism. They refer to pacemaker and follower modules to explain endogenous rhythm generation in these circuits (right vertical arrow and $\mathrm{P}$ and F nodes; same architecture as in Fig. 1). When studying circuits in the cortex, researchers can work on the cognitive patch when referring to microcircuit elements that are operative in behaviorally relevant information processing (left vertical arrow, E and I nodes), or they can work on the infrastructural patch when referring to elements that prevent damage within the circuit (middle vertical arrow, C node). ${ }^{12}$ The two patches overlap because each infrastructural function is mechanistically coupled to the cognitive function(s) it enables (left curved arrow). Conversely, analyses of cognitive circuit functions depend on the infrastructural patch (blue line piercing through the patch). Cognitive analyses rely on a functionally neutral description of CMC infrastructure, and knowledge of infrastructural functions constrains which cognitive analyses are biologically plausible.

\footnotetext{
${ }^{12}$ The fact that cortical microcircuits as a whole execute both cognitive and infrastructural functions makes them multifunctional structures (McCaffrey 2015). I discuss the issue of multifunctionality further in the conclusion.
} 
Cognitive and subsistence patches also overlap because I use the graded notion of causal specificity to distinguish cognitive and noncognitive functions. I focused on clear cases of each kind to argue that researchers need to attend to nonspecific enabling conditions to overcome cognitive myopia. Yet there are kinds of neural function whose causal specificity lies between operative and enabling conditions for cognition and behavior. ${ }^{13}$ Like with the case of nylon in the patchwork of "hardness", researchers may use empirical and conceptual tools from multiple patches to study these and other intermediate cases of "neural function". For instance, researchers may have to work at the intersection of subsistence and cognitive patches when studying pacemaker modules that are part of an information processing mechanism. Such CPG modules may not be mechanistically decoupled from cognitive functions in the way known CPGs are (upper curved arrow, right). By making the mechanistic and evolutionary relations between different kinds of neural function explicit, a patchwork approach helps researchers to decide which conceptual and empirical tools are appropriate to investigate a given mesoscale circuit. Such an approach moves beyond cognitive myopia because it shows that cognitive neuroscientific methods and information processing vocabulary occupy only one patch in the patchwork structure of "neural function". These empirical and conceptual tools are inappropriate when researchers study other, noncognitive kinds of function.

To understand the advantage of a patchwork approach over a cognitively myopic picture of "neural function", consider the function of unknown CPG circuits in the neocortex. Yuste et al. (2005, p. 481) conclude from biophysical similarities between spinal cord and cortical circuits that the cortex could contain "memory and learning" CPGs. This proposal assumes that the function of cortical CPG circuits should be evaluated with conceptual and empirical tools from the cognitive patch. As a result of cognitive myopia, however, Yuste and colleagues overlook the possibility of evaluating the function of cortical CPG circuits by combining tools from the subsistence and infrastructural patch. According to this proposal, oscillating CPG circuits in the neocortex would maintain and repair infrastructure, rather than process behaviorally relevant information. Besides overlooking noncognitive functions of cortical circuits, cognitive myopia also leads these researchers to misevaluate the evolutionary relationship between subsistence and cognitive functions. The patchwork approach distinguishes the encephalization of subsistence functions for infrastructural maintenance from their exaptation for behaviorally relevant information processing (curved arrows, top left and

\footnotetext{
${ }^{13}$ An example is the effect of global changes of neural gain on attention and learning (Eldar et al. 2013). Unlike the enabling condition of local gain control, it has a graded influence on task performance. Unlike operative conditions such as FEF eye gaze control, however, it affects many cognitive tasks at once.
} 
bottom right). Yuste et al. (2005, p. 477) simply lump these two distinct processes under the label "encephalization". This example illustrates why a patchwork approach allows researchers to evaluate the mechanistic organization and evolutionary history of neural circuit functions correctly, whereas cognitive myopia does not.

Like the circuit diagrams from sect. 3, the patchwork model of "neural function" is a conceptual template to be used in and revised by further empirical research. The taxonomy of circuit functions it provides is tailored to the purpose of understanding how information processing and maintenance/repair mechanisms in neural systems are related to each other. If neuroscientists and philosophers aim to analyse other kinds of circuit function, they can add further patches or relations between patches, as well as modify the types of circuit architecture to which the patches refer. The promise of adopting a patchwork approach is that it helps us to overcome cognitive myopia by providing the conceptual tools to describe each kind of neural function on its own terms, rather than forcing it into inappropriate vocabulary.

\section{Conclusion}

If neuroscientists and philosophers want to move beyond cognitive myopia and meet the brain on its own terms, they need to add noncognitive patches such as "subsistence function" and "infrastructural function" to their conceptual repertoire. They can use these patches to explore unknown entities and activities, rather than shoehorning them into cognitive vocabulary or discarding them as "noise" or "epiphenomena". A patchwork approach to "neural function" helps researchers to better understand how these different kinds of circuit function are mechanistically and evolutionarily related to each other. By providing tools to study both cognitive and noncognitive kinds of function, such an approach also helps researchers to explain how the brain's capacities as an information processing device are constrained by its ability to maintain and repair itself as a physiological apparatus. A patchwork approach helps neuroscientists to pursue research avenues that cognitive myopia prevents them to explore. To demonstrate the broader significance of this approach, let me conclude by sketching its application to other fields in neuroscience and its philosophy.

In neuroimaging, task-independent fluctuations measured during the experimental "resting state" can refer to endogenous brain activity with different kinds of functional roles in behavior and cognition. A patchwork approach can help to classify these kinds. Endogenous 
activities with homeostatic functions such as energy production or waste disposal are causally nonspecific enabling conditions for cognition and behavior (Raichle 2015, Kiviniemi et al. 2016). Other endogenous activities serve modulatory functions, because they affect cognition and behavior in a graded but task-unspecific fashion. Fluctuations in cortical excitability and arousal are an example of such intermediate effects (Chang et al. 2016). Yet other kinds of endogenous brain activity are likely operative in overt behavior or cognitive operations (Fox et al. 2007, Smallwood et al. 2016). Because a cognitively myopic view on resting state research focuses on operative roles only, it risks overlooking, discarding or misevaluating kinds of endogenous brain activity with homeostatic or modulatory functions. Philosophers have overlooked the potential of resting state research to study these noncognitive and intermediate functions, because they have exclusively focused on its use to study cognitive functions (Bechtel 2013, Klein 2014, McCaffrey and Danks 2017). A patchwork approach can overcome this cognitively myopic focus by keeping the enabling, modulatory and operative roles of endogenous brain activities in view at once.

Similar to work on resting-state neuroimaging, philosophical accounts of multifunctionality have overlooked noncognitive types of multifunctionality. For example: noncognitive CPG circuits can be multifunctional because they are capable of switching between different rhythmic outputs (Brigmann and Kristan 2008). Cortical microcircuits are multifunctional because they execute both information processing and infrastructural functions. By including subsistence and infrastructural patches, a patchwork approach allows scholars to characterize these noncognitive or combined forms of multifunctionality. Pace Rathkopf (2013), a patchwork approach holds that these conceptual patches complement, rather than replace cognitive concepts of function to tackle multifunctionality. A patchwork approach furthermore extends existing accounts of multifunctionality. It adds coupling and decoupling as noncognitive types of mechanistic organization to McCaffrey's typology of multifunctional structures (2015, Table 1). It also adds neuromodulation and physiological circuit properties as noncognitive kinds of context to Burnston's contextualist approach to multifunctionality (Burnston 2016). Although these arguments will have to be developed in detail elsewhere, the examples of resting-state research and multifunctionality show that the patchwork approach can help neuroscientists and philosophers to overcome cognitive myopia in a variety of topics surrounding the concept of neural function. 
Acknowledgments: I would like to thank Daniel, Burnston, David Colaço, Carl Craver, Lena Kästner, Joseph McCaffrey, and Mark-Oliver Casper as well as two anonymous reviewers for helping me to clearly articulate the ideas in this paper. I thank participants of the Neural Mechanism Online Seminar, the ISHBB conference in Sao Paolo, the OHBM conference in Vancouver and members of the Research Group for Neuroanatomy and Connectivity at the MPI for Cognitive and Brain Sciences Leipzig for valuable feedback on presentations of this material. An earlier version of this paper appeared as chapter 4 of my $\mathrm{PhD}$ dissertation "Meeting the Brain on its own terms. Exploratory Concept Formation and Noncognitive Functions in Neuroscience" (Otto-von-Guericke University Madgeburg). I thank Holger Lyre and Henrik Walter for their excellent supervision of this project.

\section{References}

Abbot, L.F. \& Chance, F.S. (2005). Drivers and modulators. Progress in Brain Research 149, $147-155$.

Akagi, M. (2017). Rethinking the problem of cognition. Synthese https://doi.org/10.1007/s11229-017-1383-2.

Appelhans B.M. \& Luecken, L.J. (2006). Heart rate variability as an index of regulated emotional responding. Reviews General Psychology. 10(3), 229-240.

Bartos, M., Vida I., Jonas, P. (2007). Synaptic mechanisms of synchronized gamma oscillations in inhibitory interneuron networks. Nature Reviews Neuroscience 8(1), $45-56$.

Bechtel, W. (2008). Mental mechanisms. Philosophical perspectives on cognitive neuroscience. New York: Routledge.

Bechtel, W. \& Abrahamsen A. (2009) Decomposing, recomposing, and situating circadian mechanisms: Three tasks in developing mechanistic explanations. In H. Leitgeb and A. Hieke, (eds.), Reduction and elimination in philosophy of mind and philosophy of neuroscience. Frankfurt: Ontos Verlag (pp. 173-186).

Bechtel, W. \& Richardson B. (2010) Discovering complexity. Decomposition and localization as strategies in scientific research. $2^{\text {nd }}$ ed., Cambridge MA: MIT Press.

Bechtel, W. (2013) The endogenously active brain: The need for an alternative cognitive architecture. Philosophia Scientiae 17, 3-30.

Beutler, L., Chen, Y., Ahn, J.S., Lin, Y.-C., Essner, R.A., Knight, Z.A. (2017). Dynamics of gut-brain communication underlying hunger. Neuron 96(2), 461-475.

Bickle, J. (2003). Philosophy and neuroscience: a ruthlessly reductionist account. Dordrecht: Kluwer Academic Publishers.

Briggmann K.L. \& Kristan W.B. (2008). Multifunctional pattern-generating circuits. Annual Reviews Neuroscience 31, 271-294.

Burnston, D. (2016). A contextualist approach to functional localization in the brain. Biology \& Philosophy 31(4), 527-550.

Bursten, J. (2016). Smaller than a breadbox: scale and natural kinds. British Journal of Philosophy of Science, axw 22, https://doi.org/10.1093/bjps/axw022 
Chang K., Leopold D.A., Schölvinck M.L., Mandelkow H., Picchioni D., Liu X., Ye F.Q., Turchi J.N., Duyn J.H. (2016). Tracking brain arousal with fMRI. PNAS 113(16), 45184523.

Chang, H. (2004). Inventing temperature. Measurement and scientific progress. Oxford: Oxford University Press

Christen, M. (2006). The role of spike patterns in neuronal information processing. A historically embedded conceptual clarification. Dissertation, ETH Zürich.

Cramer N.P., Li Y. \& Keller A. (2007). The whisking rhythm generator: A novel mammalian network for the generation of movement. Journal of Neurophysiology 97(3), 2148-2158.

Craver C.F. (2007). Explaining the brain. Mechanisms and the mosaic unity neuroscience. Oxford: Oxford University Press.

Craver, C.F. (2016). Optogenetics and maker's knowledge: foundations for a theory of translational progress. Presented paper at the 25th biennial meeting of the Philosophy of Science Association, Nov 3-5 2016, Atlanta, USA.

Cao, R. (2011). A teleosemantic approach to information in the brain. Biology and Philosophy https://doi.org/10.1007/s10539-011-9292-0

Cao, R. (2014) Signaling in the brain: In search of functional units. Philosophy of Science 81(5), 891-901.

Cummins, R. (1975) Functional analysis. Journal of Philosophy 72(20), 741-765.

De Felipe, J. (1999) Chandelier cells and epilepsy. Brain 122(10), 1807-1822.

Douglas R.D. \& Martin K.A. (1991). A functional microcircuit for cat visual cortex. Journal of Physiology 440, 735-769.

Ebbesen C.L., Doron G., Lenschow C., Brecht M. (2017). Vibrissa motor cortex activity suppresses contralateral whisking behavior. Nature Reviews Neuroscience 20(1), 82-89.

Eldar, E., Cohen, J.D. Niv, Y. (2013). The effects of neural gain on attention and learning. Nature Neuroscience 16(8), 1146-1153.

Engl, E. \& Atwell, D. (2015). Non-signalling energy use in the brain. Journal of Physiology 593(16), 3417-3429:

deCharms, R.C. \& Zador, A. (2000). Neural representation and the cortical code. Annual Reviews Neuroscience 23, 613-647.

Feest, U. \& Steinle, F. (2012) (eds.). Concepts in investigative practice. Berlin: de Gruyter.

Fox M.D., Snyder A.Z., Vincent J.L., Raichle M.E. (2007). Intrinsic fluctuations within cortical systems account for intertrial variability in human behavior. Neuron 56, 171-184.

Garson, J. (2003). The introduction of information into neurobiology. Philosophy of Science 70, 926-936.

Garson J. (2011) Selected effects and causal role functions in the brain: the case for an etiological approach to cognitive neuroscience. Biology \& Philosophy 26, 547-565.

Ghose G.M. \& Freeman, R.D. (1992). Oscillatory discharge in the visual system: Does it have a functional role? Journal of Neurophysiology 68(5), 1558-1578.

Gould, S.J. \& Vrba, E.S. (1982). Exaptation - a missing term in the science of form. Paleobiology 8(1), 4-15. 
Grillner S., Markram H., De Schutter E., Silberberg G., LeBeau F.E. (2005). Microcircuits in action-from CPGs to neocortex. Trends in Neuroscience 28(10), 525-533.

Grassmann M., Vlemincx E., von Leupoldt A., Mittelstädt J.M., Van Der Bergh, O. (2016). Respiratory changes in response to cognitive Load: a systematic review. Neural Plasticity, https://doi.org/10.1155/2016/8146809.

Haueis, P. (2018). Meeting the brain on its own terms. Exploratory concept formation and noncognitive functions in neuroscience. Dissertation, Otto-von-Guericke University Magdeburg.

Haueis, P. (2014). Meeting the brain on its own terms. Frontiers in Human Neuroscience 8(815), doi: 10.3389/fnhum.2014.00815

Heinzle J., Hepp K, Martin, K.A. (2007). A microcircuit model of the frontal eye fields. Journal of Neuroscience 27(5), 9341-9353.

Hermes, D., Miller, K., Wandell, B.A., Winawer, J. (2015). Stimulus dependence of gamma oscillations in human visual cortex. Cerebral Cortex 25(9), 2951-2959.

Heeger, D.J. (1992). Normalization of cell responses in cat striate cortex. Visual Neuroscience 9, 181-197.

Homma, I. \& Masoaka, Y. (2008) Breathing rhythms and emotion. Experimental Physiology 93(3), 1011-1021.

Iaccarino, H.F., Singer, A.C., Martorell, A.J., Rudenko. A., Gao, F., Gillingham, T.Z., Mathys, H., Seo J., Kritskiy, O., Abdurrob, F., Adaikkan, C., Canter, R.G., Rueda, R., Brown, E.N., Boyden, E.S., Tsai, L.H. (2016). Gamma frequency entrainment attenuates amyloid load and modifies microglia. Nature 540(7632), 230-235.

Kandel, E.R., Schwartz, J.H., Jessell, T.M., Siegelbaum, S.A., Hudspeth, A.J. (eds.) (2012) Principles of neural science. $5^{\text {th }}$ ed., ( $4^{\text {th }}$ edition 2000) New York: McGraw-Hill.

Kiviniemi, V., Wang, X., Korhonen, V., Keinänen, T., Tuovinen, T., Autio, J., LeVan, P., Keilholz, S., Zang, Y.F., Hennig, J., Nedergaard, M. (2016). Ultra-fast magnetic resonance encephalography of physiological brain activity - glymphatic pulsation mechanisms? Journal of Cerebral Blood Flow and Metabolism 36(6), 1033-1045.

Kirschfeld, K. (1992). Oscillations in the insect brain: do they correspond to the cortical gamma waves of vertebrates? PNAS 89(10), 4764-4768.

Klein, C. (2014). The brain at rest: what it's doing and why that matters. Philosophy of Science 81(5), 974-985.

Klein, C. (2017). Brain regions as difference makers. Philosophical Psychology 30(1-2), $1-20$.

Love, A.C. (2013). Theory is as theory does. Scientific practice and theory structure in biology. Biol Theory 7(4), 325-337.

Luque-Casado, A., Zabala, M., Morales, E., Mateo-March, M., Sanabria, D. (2013). Cognitive performance and heart rate variability: the influence of fitness level. PLoS One 8(2), https://doi.org/10.1371/journal.pone.0056935

Marder, E., Goaillard, J.-M. (2006) Variability, compensation and homeostasis in neuron and network function. Nature Reviews Neuroscience 7(7), 563-574. 
Marder, E., Bucher, D.M. (2007). Understanding circuit dynamics using the stomatogastric nervous system of lobsters and crabs. Annual Reviews Neuroscience 69, 291-316.

Marder, E. (2012). Neuromodulation of neuronal circuits: back to the future. Neuron 76(1), 111.

Martin, C.B., Deutscher, M. (1966). Remembering. Philosophical Review 75(2), 161-96.

McCaffrey, J. (2015). The brain's heterogenous functional landscape. Philosophy of Science 82(5), 1010-1022.

McCaffrey, J. \& Danks, D. (2017). Mixtures and psychological inference with resting state fMRI. British Journal for Philosophy of Science, axx053, https://doi.org/10.1093/bjps/axx053

Merker, B. (2013). Cortical gamma oscillations: the functional key is activation, not cognition Neuroscience Biobehavioral Reviews 37(3), 401-417.

Mesulam M.M. (1998). From sensation to cognition. Brain 121(6), 1013-1052.

Moore, J.D., Deschênes, M., Furuta, T., Huber, D., Smear, D.C., Demers, M., Kleinfeld, D. (2013). Hierarchy of orofacial behaviors revealed through whisking and breathing. Nature 497(7448), 205-210.

Nersessian, N. (2008). Creating scientific concepts. Cambridge, MA: MIT Press.

Ni J., Wunderle, T., Lewis, C.M., Desimone, R., Diester, I., Fries, P. (2016). Gamma-rhythmic gain modulation. Neuron 92(1), 240-251.

Novick, A. (2018). The fine structure of ,homology'. Biology \& Philosophy 33(6) https://doi.org/10.1007/s10539-018-9617-3.

O’Leary, T., Williams, A.H., Caplan, J.C., Marder, E. (2014). Cell types, network homeostasis, and pathological compensation from a biologically plausible ion channel expression model. Neuron 82(4), 809-821.

Panzeri, S., Harvey, C.D. Piasini, E., Latham, P.E., Fellin, T. (2017). Cracking the neural code for sensory perception by combining statistics, intervention, and behavior. Neuron 93(3), 491-507.

Piccinini, G. \& Bahar, S. (2013). Neural computation and the computational theory of cognition. Cognitive Science 37(3), 453-488.

Rathkopf, C. (2013). Intrinsic function and localization. Philosophy of Science 80(1), 1-21.

Rathkopf, C. (2017). Neural information and the problem of objectivity. Biology \& Philosophy $32,321-336$.

Raichle, M.E. (2015). The Restless brain. How intrinsic activity organizes brain function. Philosophical Transactions of the Royal Society London 370(1668), https://doi: 10.1098/rstb.2014.0172.

Rieke F., Warland D., de Ruyter van Steveninck R.R., Bialek W. (1999). Spikes: exploring the neural code. 2nd ed., Cambridge, MA: MIT Press.

Rouse, J. (2015). Articulating the world. Conceptual understanding and the scientific image. Chicago: Chicago University Press. 
Selverston, A.I. (2010). Invertebrate central pattern generator circuits. Philosophical Transactions of the Royal Society London B 365(1551), 2329-2345.

Silva, A.J., Landreth, A., Bickle, J. (2013). Engineering the next revolution in neuroscience. The new science of experiment planning. Oxford: Oxford University Press.

Smallwood, J., Karapanagiotidis, T., Ruby, F., Medea, B., de Caso, I., Konishi, M., Wang, T., Hallam, G., Margulies, D.S., Jeffries E. (2016). Representing representation: integration between the temporal lobe and the posterior cingulate influences the content and form of spontaneous thought. PLoS ONE 11(4), https://doi.org/10.1371/journal.pone.0152272.

Squire, L.R., Berg, D., Bloom, F.E., du Lac, S., Ghosh, A., Spitzer, N.C., (eds.) (2012) Fundamental neuroscience. $4^{\text {th }}$ ed., Academic Press, Amsterdam.

Sreenivasan, V., Karmakar, K., Rijli, F.M., Petersen, C.C.H. (2014). Parallel pathways from motor and somatosensory cortex for controlling whisker movements in mice. European Journal of Neuroscience 41(3), 354-367.

Stevens, C.F. (1996) Spatial memory: the beginning of a dream. Cell 87(7), 1147-1148.

Šustar, P. (2007). Neo-functional analysis: phylogenetic restrictions on causal role functions. Philosophy of Science 74(5), 601-615.

Wilson, M. (2006). Wandering Significance. An essay in conceptual behavior. Oxford: Clarendon Press.

Woodruff, A.R., Anderson, S.A., Yuste, R. (2010). The enigmatic function of chandelier cells. Frontiers in Neuroscience 4(201), https://doi.org/10.3389/fnins.2010.00201.

Woodward, J. (2010). Causation in biology: stability, Specificity and the choice of levels of explanation. Biology \& Philosophy 25(3), 287-318.

Yuste, R., MacLean, J., Smith, J., Lansner, A. (2005). The cortex as a central pattern generator. Nature Reviews Neuroscience 6(6), 477-483.

Zhu, Y., Stornetta, R.L., Zhu, J.J. (2004). Chandelier cells control excessive cortical excitation: characteristics of whisker-evoked synaptic responses of layer 2/3 Nonpyramidal and pyramidal neurons. Journal of Neuroscience 24(22), 5101-5108. 